\title{
Bee Products Quality Control and Emergency Management Mechanism Research Based on Multi-Agent
}

\author{
E. Yue ${ }^{1}$, Yongsheng $\mathrm{Cao}^{2}$, and Yeping $\mathrm{Zhu}^{1}$ \\ ${ }^{1}$ Research Institute of Agriculture Information, \\ Chinese Academy of Agricultural Science, \\ Key Laboratory of Digital Agricultural Early Warning Technology \\ of Ministry of Agricultural, Beijing, China, 10008 \\ eyue@mail.caas.net.cn \\ ${ }^{2}$ Institute of Crop Science, Chinese Academy of Agricultural Science, \\ Beijing, China, 10008
}

\begin{abstract}
Based on the characteristics about distribution, intelligent, and coordination of Multi-Agent system, In this article, Agent theory and technology is applied to bee products quality control and emergency management system of information interaction and coordination, we constructed the bee products quality control and emergency management system of the frame structure, and put forward bee products quality control and emergency management system of the blackboard communication mechanism, and used the blackboard structure on the bee products quality control and emergency decision mechanism for research.
\end{abstract}

Keywords: Multi-Agent, Bee products, quality control, emergency management, communication mechanism, distributed blackboard structure Maize, Purity identification, Density, Color, Core area.

\section{Introduction}

Bee products quality control and emergency management can to provide timely, quick, efficient, high quality emergency services for our country bee products quality control department when there are many quality problems, or natural disaster situation. To the question about Bee products quality problem, all kinds of emergencies independence and the correlation, need many departments and units of collaborative and cooperate in emergency decision and rescue that process. Bee products quality control and emergency management system must know accident of real-time information, and more effective interaction for rapid comprehensive decision-making system.

However, China's bees industry in the information, intelligence bee products quality control and emergency management has just starting, emergency response and transfer mode the lack of effective information release mechanism, use of not enough real-time information and feedback information, Department of the different response system, information platform and software platform lead to share data, response and scheduling model is not the same. All of these lead to the emergency rescue operation can't efficient conduct. With the rapid development of information technology, 
network has entered into the public's daily life and work, therefore, we can use of the distributed technology based on the network to construct distributed emergency response system, MAS because its have distribution, expansibility, parallelism, flexibility, intelligence and so on, the more suitable for bee products quality control and emergency management system construction.

MAS (Multi-Agent System) is a system concept on the basis of the Distributed Artificial Intelligence (Distributed Artificial Intelligence, DAI)[1]. Each Agent has the ability about independently solving problems or independently finished function, each Agent through information interaction, negotiated, coordinate complete complex task, Agent can be parallel to transmission and control information. However, in distributed open environment, each Agent is often heterogeneous, dynamic and unpredictable, it has the distribution and the internal correlation, how to through the communication mechanism to realize more effective interaction is a key issue. we must through the interaction between the Agent to realize resource sharing, the coordination of the conflict in order to achieve overall goal.

In this article, based on the characteristics about distribution, intelligent, and coordination of Multi-Agent system, Agent theory and technology is applied to bee products quality control and emergency management system of information interaction and coordination, we constructed the bee products quality control and emergency management system of the frame structure, and put forward bee products quality control and emergency management system of the blackboard communication mechanism, and used the blackboard structure on the bee products quality control and emergency decision mechanism for research. For bee products quality control and emergency management to provide an effective research ideas and methods.

\section{Multi-Agent Communication Mechanism}

The communication mechanism is Agent that in what ways does realize communication. Many of the Agent communication mechanism not only to realize the bottom of communication between the computer, but also to run in different network platform for communications connection, Agent in the environment of networks which position can be effective play its function and other collaborative interaction Agent. The communication mechanism design and choose plays an important role in between maintaining cooperation, to solve the conflict and to realize information exchange between agent, it will directly affect the overall efficiency of the system, robustness and expansibility.

At present, many of the main communication Agent system has there kinds: blackboard, federation, peer-to-peer $[2,3,4]$.

\subsection{The Blackboard}

The blackboard is actually a shared knowledge structure, it for each Agent provides a share data area, used in between Agent of information and data exchange and sharing. Each Agent can write directly to the blackboard information, also can from the blackboard read other Agent of information. Allow many Agent through to the blackboard content directly to read and write to distribute and get news [5], it could achieve a send perform multiple tasks of communication. In the central control system, the blackboard way can share data structure and realizing efficient communication. The blackboard has advantages 
during the central control and high efficient, yet for complexity, interaction, and safety requirements high task, a blackboard way has some limitations.

\section{2 $\quad$ Federation}

All members of agent constitute different groups on the basis of federation, each Agent group set a Agent medium, Each Agent only communication with in the group media Agent, media Agent according to the request of the Agent, the news sent to the agent in group. This method greatly reduces the direct communication link between Agent, but if the number of Agent less, frequent communication between Agent, through the media Agent communication efficiency but is not high.

\subsection{Peer-to-Peer}

A peer-to-peer using TCP/IP protocol, establish the communication is the direct physical connection link. The packet to ensure the safe arrival, for TCP/IP protocol can realize the end-to-end confirmation. At present, most of the Agent platform established using direct communication mode. Using a peer-to-peer realize the physical connection between Agent for the sender Agent must know the receiving Agent physical location in the system.

\section{Bee Products Quality Control and Emergency Management Communication Mechanism Based on the Agent}

The core of Bee products quality control and emergency management is integration information resources and emergency management resources about our country different management and testing department, to achieve the emergency coordination effect between different departments, On the whole, enhance the system of the emergency response and decision making. Multi-Agent system has many good properties, it more suitable for the construction of bee products quality control and emergency management system. This section we will be structure bee products quality control and emergency management frame structure based on Agent, and use the Agent communication coordination technology research bee products quality control and emergency management system of communication mechanism.

\subsection{Bee Products Quality Control and Emergency Management System Structure Based on Agent}

Bee products quality control and emergency management system based on Agent, each Agent is real-time and autonomy basic modules, each Agent mutual cooperation and coordination, and compose a real-time function entity.

In the bee products quality control and emergency management system, Bee products quality control and emergency management organizations included ministry of agriculture, the national testing center, weather and environmental protection department and so on. They assume the bee products quality control and emergency management tasks. Every department is independent agent, and each main department Agent included many other auxiliary function agent, such as implementation agent and monitoring agent and so on. In the bee products quality control and emergency management organization, in order to better coordinate emergency management, optimize emergency management 
resources, to achieve the effective emergency decision knowledge together, we set up an emergency management center agent, the emergency management center agent include Central agent and Local agent, Every province is a Local agent, these agents jointly responsible for bee products quality control and emergency management in the system.

In figure 1 description of the typical distributed bee products quality control and emergency management structure based on Multi-Agent. Central management Agent in the global scheduling and decision-making position, then, according to China's bees products feature, The local agent of the province mainly complete emergency management functions (such as: daily management, emergency command). When need resources sharing and information collaboration between province, each of local Agent through communication network and information platform for exchange information. Central Agent plays a comprehensive coordination and decision-making role, integration and coordination each local Agent task.

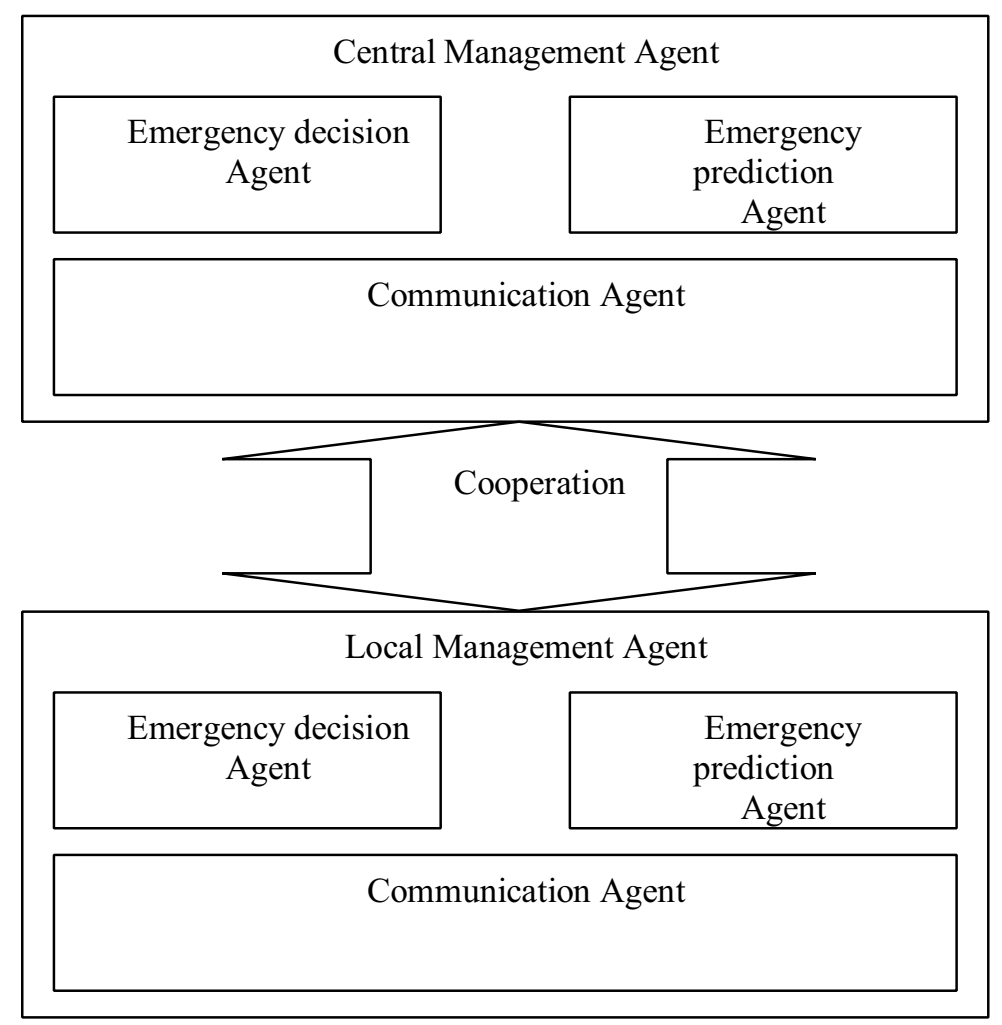

Fig. 1. Bee products quality control and emergency management structure based on Multi-Agent

\subsection{Emergency Management Communication Mechanism Based on Agent}

Different local Agent distribution in different environment, Heterogeneous platforms and information produced some obstacles to information of interaction and coordinate. In order to promote the cooperation between local Agent, to realize the integration of 
heterogeneous knowledge, in this paper, we use distributed blackboard structure to describe emergency management of communication coordination mechanism. Distributed blackboard structure can provide concurrency control, interactive, real-time control etc.

Among them, each Agent are adopted the blackboard to transfer information form the communication interface between Agent, enhance the efficiency about cooperation and communication between Agent. Every function agent has a blackboard, the blackboard is communication and information storage area, and is an information communication interface channel with other agent. At the same time, central agent and local agent have emergency blackboard, accept and response to each function agent of information, and send coordination and decision-making information to function agent.

In the process, there is two tasks collaborative request response mechanism.

\subsubsection{Cooperative Mechanism between Regional}

When department Agent can't alone finish emergency task, it through the blackboard structure to the local Agent sends a work request, if the local Agent think the request need other agent collaborative, the local agent release information on the blackboard, At the same time, the local agent analysis the task, send out task collaborative request to corresponding function agent, corresponding function agent received collaborative request, according to oneself circumstance to provide the service. Communication Agent is a control unit, to a certain extent, to realize department Agent communication request, equivalent to router, as shown in figure 2 shows.

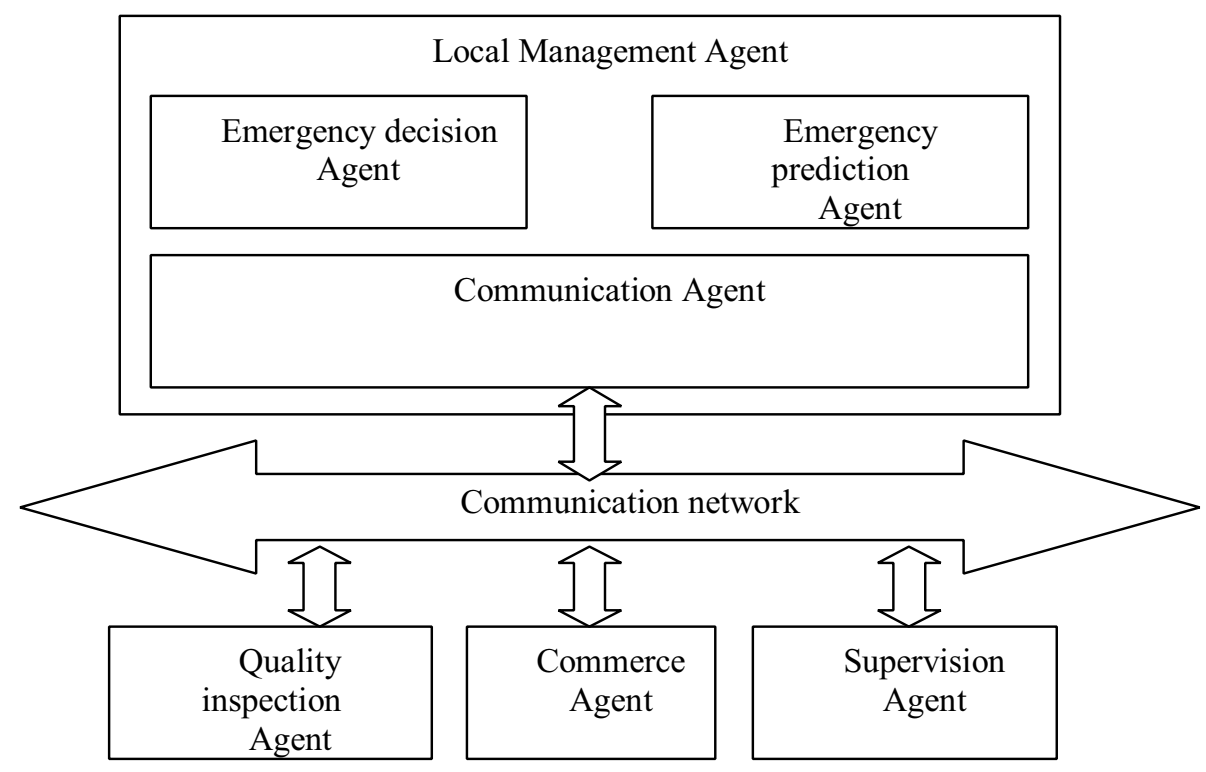

Fig. 2. Cooperative mechanism between regional

\subsubsection{Overall Situation Decomposition Collaborative Mechanism}

Central Agent establish an emergency task decomposition and task execution situation evaluation, first, overall emergency task decomposition, and assigned to each local agent, 
and then, appraise task execute condition, to more control effectively scheduling. The difference between overall situation decomposition collaborative mechanism and cooperative mechanism between regional, Central Agent monitoring the emergency blackboard information, if find local Agent is not able to accomplish task, must transregional information coordination, at this time, central agent control all emergency task, and then task decomposition, release the task decomposition to the local Agent, this is a cross-regional emergency "big synergy". Due to cooperative mechanism between regional, First, local agent accept department agent collaborative request, then build synergy between department Agent, and execute emergency task, as shown in figure 3.

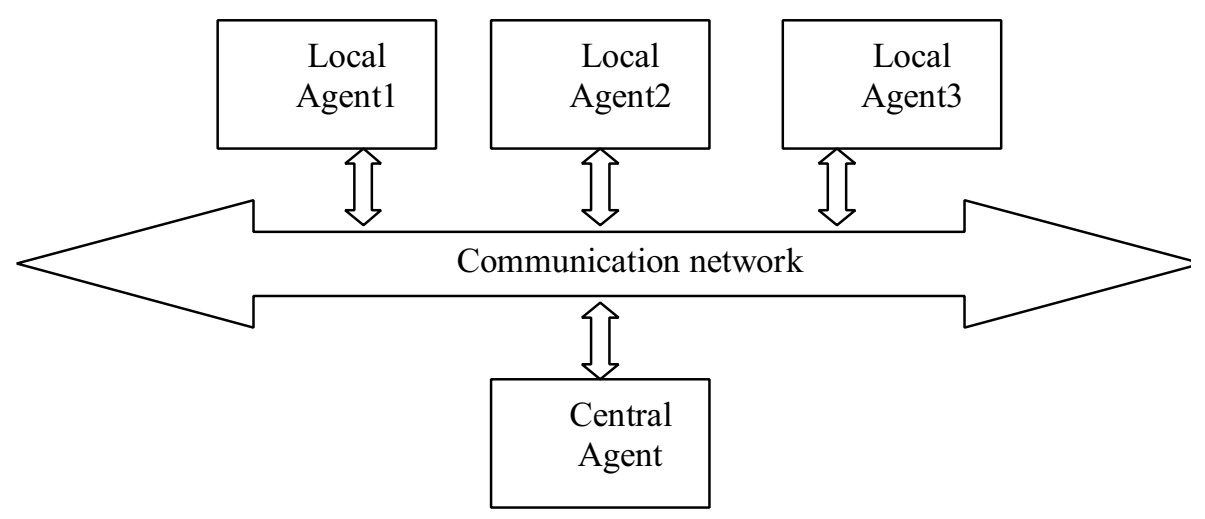

Fig. 3. Overall situation decomposition collaborative mechanism

In short, in the emergency management system, Through emergency blackboard complete information transmission and interactive between each Agent, and in different situation use different cooperative mechanism, through the blackboard complete information for the sharing and exchange, and finally realize the emergency management system of the effective information synergy.

\section{Bee Products Emergency Management Mechanism Based on Agent}

The basic thought of the emergency management is based on the blackboard structure as problem solving mechanism, the Multi-Agent technology as a problem solving way. Agent will emergency decision problem solving process simulation for mathematical model, knowledge model and the man's judgment package unified format, and form problem solving knowledge module, in intelligent solving process, their external behavior is consistent, behavior act on their own and environment, and can respond to the environment, through operation the blackboard, and cooperating problem solving. 
Emergency decision simulation intelligent blackboard model consists of three parts: the Emergency blackboard, the Emergency decision Agent, Emergency prediction Agent, shown in figure 4.

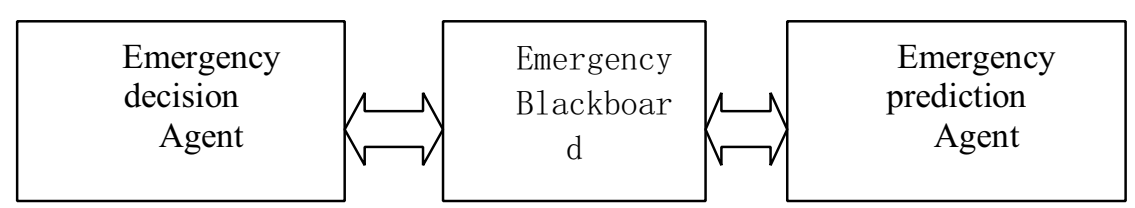

Fig. 4. Emergency decision simulation intelligent blackboard model

\subsection{Emergency Blackboard}

Emergency blackboard is a global database, it store the original data, problem solving solutions. In Emergency decision, Emergency decision information defined as a group of variables, according to the variable physical meaning divided into two categories: state variables, events variables.

(1)State variables: included production data, processing data, testing data, geography data, climate data, and so on. The variable is a function of time, the value with the advancement of crisis changes, it Can be a $(0,1)$ between the quantitative value digital.

Events variables: Defined as all events in the process of the crisis happened; the variable is a function of time, the value with the advancement of crisis changes, it Can be a $(0,1)$ between the quantitative value digital.

The emergency information of emergency simulation through state variables to reflect.

The emergency participants perceive the current crisis through emergency state variables, and decision making based on state variables. Decision making behavior of participants to display a series of events, the occurrence of events led to the change of state variables, and promote the development of the crisis. Otherwise, even if no decision action, the state variables will still be the advancement of change over time. So decision action and time is direct reason to promote action and situation change. The situation is interaction between variables, the mutual influence, part of the trend of the change will cause other variable situation of a series of variable chain reaction.

\subsection{Emergency Decision Agent}

Emergency decision Agent mainly complete crisis situations of the situation analysis, risk assessment, strength analysis, scheme generation, scheme evaluation and so on. the operation modes is when the state variables change up to a certain value, and the value(between 0-1) match crisis decision Agent executive premise, the Agent will be motivated to perform some action. For each one of the Emergency decision work, Emergency decision to realize the function of the Agent essence of a crisis situation analysis, decision making, evaluation model. 


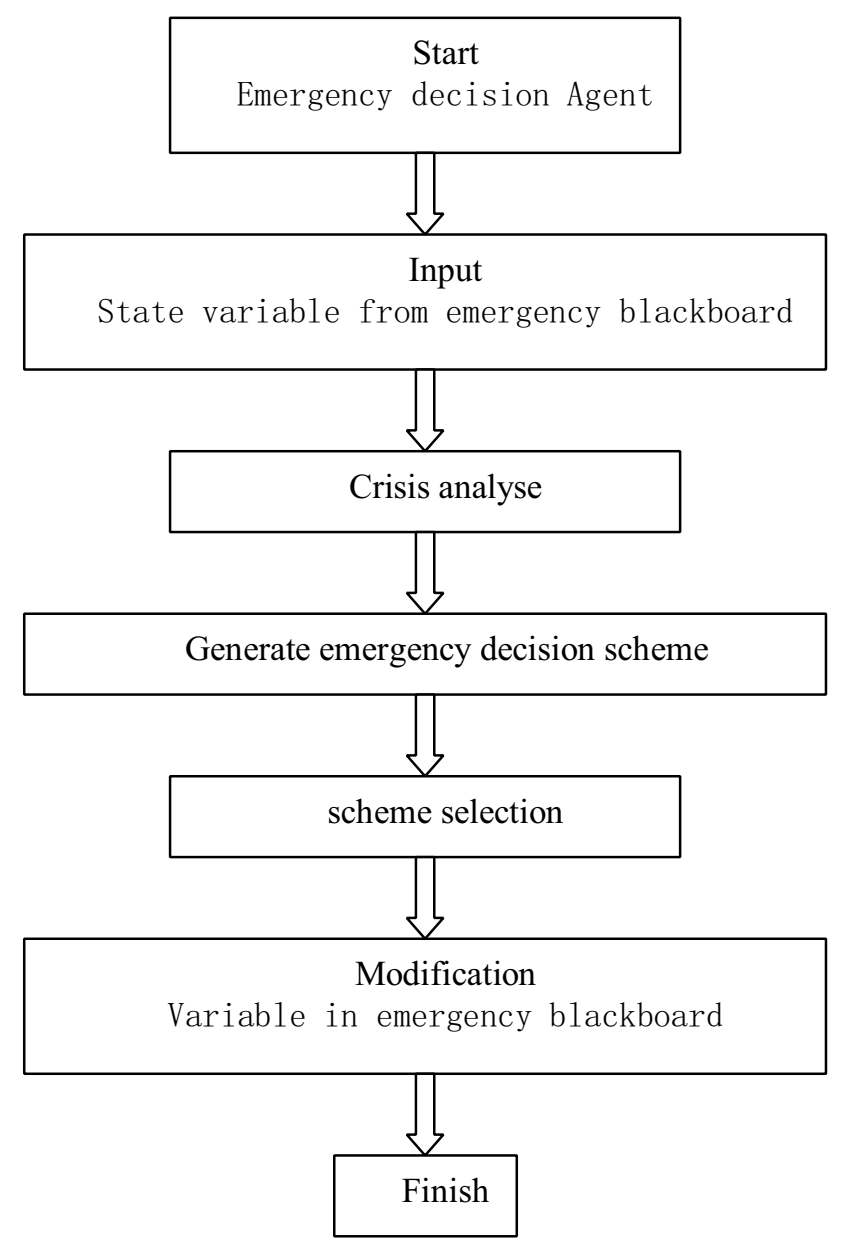

Fig. 5. Emergency decision Agent activity diagram

\subsection{Emergency Prediction Agent}

Emergency prediction Agent main complete crisis analysis of results, crisis situation variables revision and so on, the operation modes is when the situation variables change up to a certain value, and the value(between 0-1) match crisis decision Agent executive premise, the Agent will be motivated to perform some action. For each one of the Emergency decision work, Emergency prediction to realize the function of the Agent essence of Crisis result analysis, variable modification, to understand that a self-learning model. 


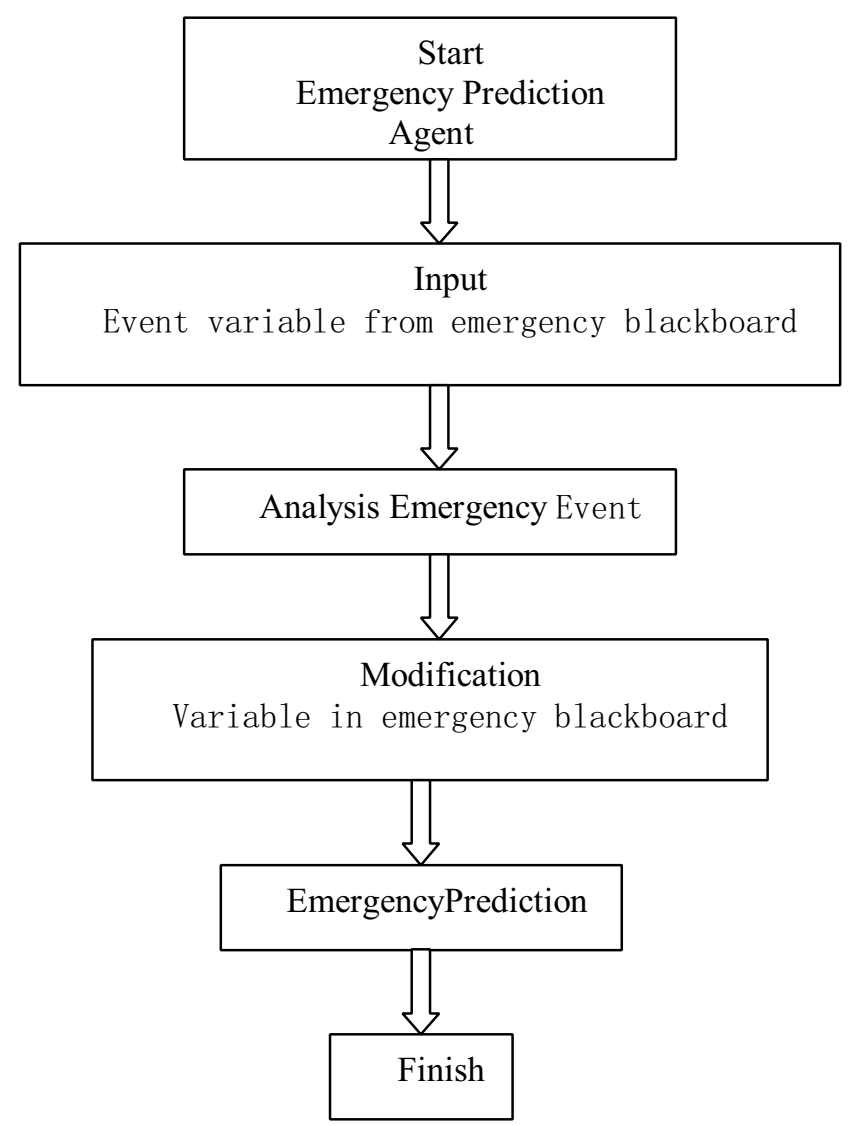

Fig. 6. Emergency prediction Agent activity diagram

Through the argument that, each Agent by conditions and action of two parts, the condition is the use of Agent premise, is a set of information about the blackboard changes of judgement, action description of the operation about the Agent usually process. They are the whole emergency decision core part, they can understand crisis in time of the current state changes, can to the change of state to make timely response ability, to have Reasoning and prediction capability through the reasoning mechanism based on the knowledge and experience, to can response crisis situation change and independent judgment in no manual intervention case.

\section{Conclusion}

With the fast development of the economy, bee products quality security is increasingly prominent, bee products related administrative departments for emergency management needs become very urgent. Considering the emergency management system of the distributed and autonomy characteristics, in this paper, Agent theory and technology is 
applied to bee products quality control and emergency management system of information interaction and coordination, we constructed the bee products quality control and emergency management system of the frame structure, we put forward bee products quality control and emergency management system of the blackboard communication mechanism, and used the blackboard structure on the bee products quality control and crisis decision mechanism for research. The method provides a good strategy for emergency management resources scheduling and the implementation of the emergency measures, to provide strong support, to improve emergency management level, ensure the bee products quality security and stability.

In the future, we need to be further research bee products quality control and urgent management resources synergy optimization mechanism based on the communication mechanism collaborative optimization, to realize information resources and emergency management resources of emergency interact and collaborative allocation in the emergency management.

Ackonwledgements. This research was supported by National Scientific and Technical Supporting Programs Funded by Ministry of Science and Technology of China (nyhyzx07-041). National Natural Science Foundation about Agent-based quality control of agricultural products (60972154).

\section{References}

1. Yao, L., Zhang, W.M.: Intelligent and Cooperative Information Systems. Publishing of Electronics Industry, Beijing (2008)

2. Luger, G.F.: Artificial intelligence. Publishing of Electronics Industry, Beijing (2008)

3. Wang, L., Zhang, Y.: Key Techniques to Realize Cooperation of Mobile Agent in MAS Environment. School of Computer Science and Engineering 32(2), 158-163 (2009)

4. Trappey, A.J.C., Trappey, C.V., Hou, J.-L.: Mobile agent technology and application for online global logistic services. Industrial Management \& Data System 104(1/2), 169-183 (2008)

5. Zhao, J.M., Zhuang, X.P.: An agent-oriented requirement analysis and modeling method 4, 33-35 (November 2009)

6. Zhao, J.: Bee product quality and safety analysis of the key technology research and development. China Apiculture 57(12), 30-32 (2006)

7. DeLoach, S.A.: Engineering Organization-Based Multiagent Systems. In: Garcia, A., Choren, R., Lucena, C., Giorgini, P., Holvoet, T., Romanovsky, A. (eds.) SELMAS 2005. LNCS, vol. 3914, pp. 109-125. Springer, Heidelberg (2006)

8. Chang, M.-H., Harrington Jr., J.E.: Agent-based models of organizations. In: Handbook of Computational Economics II (2006)

9. Yi, S.C.: Computing based on Agent. Publishing of Tsinghua University (2007)

10. Zhi, S.Z.: Advanced Artificial Intelligence. Publishing of Science (2006) 